\title{
WLTP Demands Better Aerodynamics
}

\section{Dear Reader,}

The issues relating to RDE and WLTP are very complex, as a considerable role is played in real driving situations by such factors as environmental conditions, the route driven, the driving style and the fuel used. Due to the huge amount of excess power available in most cars, the challenges resulting from legislation on $\mathrm{CO}_{2}$ emissions and air quality can only be solved systemically in interaction between the powertrain and the complete vehicle.

The indication of so-called worst case $\mathrm{CO}_{2}$ emission levels will be mandatory, and individual $\mathrm{CO}_{2}$ levels for single vehicles and variants are permitted. As a result, optional equipment will have to be optimised. A longer cycle duration, faster acceleration and a higher speed represent a much more realistic driving cycle. Consequently, the influence of electrical components decreases, whereas the contribution made by aerodynamic drag - and therefore the relevance of aerodynamics in vehicle development - will significantly increase. If one assumes that the recuperation capability of vehicles will rise to $50 \%$ due to WLTP, the contribution made by aerodynamics across all vehicle classes will increase by around 10 to $12 \%$. This means that optional equipment will have to be evaluated more accurately in future, that more time and effort will be required in coast down tests, that more families will be created within a vehicle type and substitute tests in the wind tunnel will be unavoidable.

As many customers will probably not want to give up their much-loved optional extras, there is greater focus on such things as wheels, add-on parts and even cooling systems. A whole bun- dle of measures for drag reduction will therefore be necessary. These include, for example, the optimisation of airflow around the wheels, under the floor and around the exterior mirrors, as well as a special design of the outer panels and on-demand guidance of cooling air. Active aerodynamics measures such as active spoilers and ride-height control systems may also find their way into mid-size and smaller vehicle categories in the future.

Changes in social values mean that, in future, one of the decisive criteria for success or failure will be the size of the ecological footprint of a vehicle and therefore of a brand. Customers may soon be offered such information even when they are configuring their new car.

Best wishes,

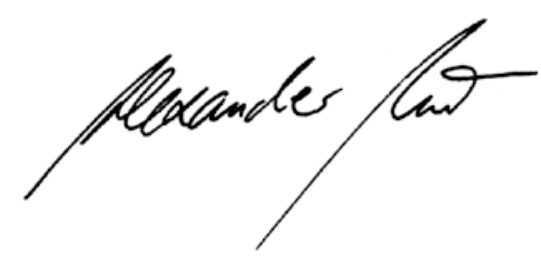

Dr. Alexander Heintzel

Editor in Chief

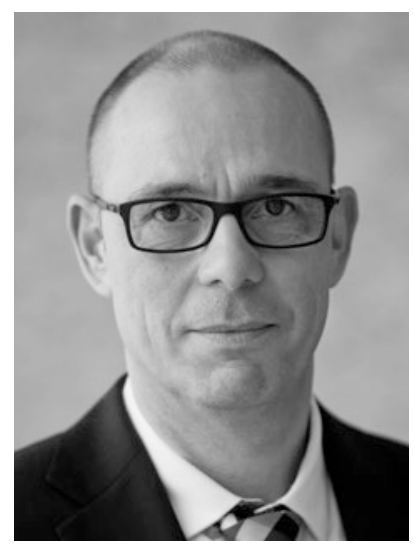

